\section{CARACTERIZACIÓN AGROMORFOLÓGICA DE MUESTRAS DE TOMATE DE OAXACA}

\section{AGROMORPHOLOGICAL CHARACTERIZATION OF TOMATO SAMPLES FROM OAXACA}

\author{
José C. Carrillo Rodríguez ${ }^{1}$ \\ y José L. Chávez Servia ${ }^{2 *}$
}

\begin{abstract}
${ }^{1}$ Instituto Tecnológico del Valle de Oaxaca, Ex-Hacienda Nazareno. 71230, Santa Cruz Xoxocotlán, Oaxaca, México. ${ }^{2}$ Centro Interdisciplinario de Investigación para el Desarrollo Integral RegionalInstituto Politécnico Nacional Unidad Oaxaca, Hornos \# 1003. 71230, Santa Cruz Xoxocotlán, Oaxaca, México.
\end{abstract}

* Autor para correspondencia (jchavezservia@syahoo.com)

\section{RESUMEN}

Los programas de mejoramiento de tomate generalmente manejan una base genética estrecha, por lo que requieren explorar y conocer la variación genética natural como fuente de genes para mejorar la productividad, adaptación y resolver problemas de plagas y enfermedades. Con el objetivo de caracterizar la variación agromorfológica de una colección de tomate silvestres y semidomesticados (Lycopersicon esculentum var. cerasiforme Dunal) del Estado de Oaxaca, México, se hizo una colecta y caracterización en invernadero de 49 poblaciones. Se detectaron diferencias significativas entre colectas en todos los caracteres evaluados, excepto en días a inicio de fructificación del quinto racimo. El análisis de componentes principales mostró que las variables días a inicios de floración, fructificación y maduración, y número total de frutos al quinto racimo, altura de planta a $30 \mathrm{~d}$ del trasplante, número de frutos por racimo y peso medio de frutos fueron las de mayor valor para describir la variabilidad morfológica. En el análisis de conglomerados se formaron dos grupos, uno de tomates semidomesticados y otro de silvestres. El primero se integró por cuatro subgrupos de variantes con frutos de forma periforme o achatada con hombros de 3 a 5 lóculos, diámetros de 2.5 a $3.7 \mathrm{~cm}$ y longitudes de 2.6 a $3.1 \mathrm{~cm}$. El segundo grupo reunió a las poblaciones de frutos más pequeños, indicativo de su condición silvestre, con frutos de dos lóculos, diámetros y longitudes menores a $2 \mathrm{~cm}$, alta producción de flores por racimo (5 a 7) y de 20 a 30 frutos al quinto racimo, precocidad a la fructificación del quinto racimo (53 a 55 d después del trasplante) y crecimiento indeterminado ( 1.3 a $1.7 \mathrm{~m}$ a $60 \mathrm{~d}$ del trasplante). En Oaxaca existe una amplia variación en caracteres fenológicos, de planta, tallo, hoja, flores y frutos en las poblaciones silvestres y semidomesticadas de tomate.

Palabras clave: Lycopersicon esculentum, análisis de conglomerados, componentes principales, variación fenotípica.

\section{SUMMARY}

Tomato breeding programs generally manage a narrow genetic base, and thus it is necessary to explore and identify the natural genetic variation of this specie as a source of genes for improving productivity, improving adaptation, and solving pest and disease problems. To characterize the agromorphological variation of a collection of both wild and semidomesticated tomatoes (Lycopersicon esculentum var cerasiforme Dunal) from the state of Oaxaca, México, 49 populations were collected and characterized under greenhouse conditions. Significant differences among populations were detected in all evaluated traits except for days to fruiting on the fifth raceme. Principal component analysis showed that days after transplant to blossom initiation, fructification ripening, number of fruits on the fifth raceme, plant height at $30 \mathrm{~d}$ after transplant, fruits per raceme, and average fruit weight were the most important traits for describing morphological variability. Cluster analysis revealed the existence of two large groups, one for the wild and another for the semi-domesticated tomatoes. The first group consisted of four subgroups of variants with pear-shaped or rounded-flattened fruits with 3 to 5 locules, 2.5 to $3.7 \mathrm{~cm}$ in diameter and 2.6 to $3.1 \mathrm{~cm}$ in length. The second group joined populations with smaller fruits, indicating their wild condition, fruits with two locules, fruit diameter and length less than $2 \mathrm{~cm}$, high production of flowers per raceme (5 to 7) and 20 to 30 fruits at the fifth raceme, precocity for fruiting at the fifth raceme (53 to $55 \mathrm{~d}$ after transplant) and indeterminate growth $(1.3$ to $1.7 \mathrm{~m}$ at $60 \mathrm{~d}$ after transplant). There is important variation in Oaxaca regarding phenological, plant, stem, leaf, flower and fruit traits in both wild and semi-domesticated populations of tomato.

Index words: Lycopersicon esculentum, cluster analysis, principal components, phenotypic variation.

\section{INTRODUCCIÓN}

México es un centro domesticación y diversificación del tomate rojo cultivado (Jenkins, 1948; Rick y Fobes, 1975; Peralta y Spooner, 2007). Las diversas exploraciones realizadas indican que Lycopersicon esculentum var. cerasiforme Dunal se distribuye en los trópicos, subtrópicos y regiones semi-secas del país, desde el Estado de Sinaloa (Sánchez-Peña et al., 2006) hasta la península de Yucatán (Nuez y Pico, 1997). No obstante su amplia distribución, hay poca documentación del potencial genético y su aprovechamiento directo o como fuente de genes para el mejoramiento.

Álvarez-Hernández et al. (2009) en Michoacán, identificaron diversas especies plaga asociadas al tomate silvestre, las cuales no obstruyeron la fructificación; por su parte, Sánchez-Peña et al. (2006) determinaron una menor incidencia de mosquita blanca (Bemisia tabaci) en tomate silvestre de Sinaloa que posee alta densidad de tricomas, lo que podría indicar que las poblaciones silvestres han desarrollado mecanismos de adaptación a diversas condiciones de estrés. Aquí el término silvestre se usa para referirse a las plantas que crecen en ambientes naturales, en cercas y campos de cultivo como maleza, sin 
la intervención del hombre; y el término semidomesticado se usa para identificar a las plantas que fueron de origen silvestre pero que ya son cultivadas o manejadas por el hombre y están en proceso de domesticación (Sevilla y Holle, 2004).

Los actuales programas de mejoramiento genético buscan, además de mejorar productividad y adaptación, incorporar alta calidad nutricional y resistencia a patógenos a las variedades, a través de introgresión, retrocruzamientos (Zamir et al., 1999; Schauer et al., 2006) y otros esquemas de mejoramiento. El uso de germoplasma silvestre se inició en Estados Unidos desde 1930 (Rick, 1986), pero poco se hace en México al respecto. Antes de promover su utilización en el mejoramiento del tomate, es necesario conocer la variabilidad genética que se conserva in situ. No hay reportes específicos de caracterización morfológica del tomate semidomesticado y silvestre de Oaxaca, y sólo se han reportado 20 accesiones en el banco de germoplasma de la Universidad Autónoma Chapingo-Valles Centrales (Molina y Córdoba, 2006). El presente trabajo se realizó con el objetivo de caracterizar la variación agromorfológica de una colección de 49 colectas de tomates, silvestres o semidomesticados, del Estado de Oaxaca, México.

\section{MATERIALES Y MÉTODOS}

\section{Material vegetal y evaluación agromorfológica}

Se colectaron y recolectaron 49 muestras poblacionales de semilla de tomate semidomesticado (21) y silvestre (28), en ecosistemas naturales y perturbados y en comunidades comprendidas territorialmente en 25 municipios de ocho regiones oaxaqueñas: Cañada, Mixteca, Costa, Sierra Sur, Sierra Norte, Tuxtepec y Valles Centrales, localizadas entre las coordenadas geográficas $15^{\circ} \quad 47^{\prime}$ 05'” 18 $18^{\circ} 29^{\prime} 06^{\prime \prime}$ LN y $95^{\circ} 13$ ' 32" a $97^{\circ} 56^{\prime} 06^{\prime \prime}$ LO, con variaciones de altitud de 15 a $2300 \mathrm{~m}$ (Cuadro 1). Las muestras se sembraron en invernadero durante el ciclo primavera de 2009, bajo un diseño de bloques completos al azar con cuatro repeticiones. El invernadero se ubica en la Exhacienda El Nazareno, Santa Cruz Xoxocotlán, Oaxaca. El trasplante se hizo 35 d después de la siembra. Durante el cultivo se implementó el sistema de fertirriego por goteo con las fórmulas 12-43-12, 19-19-19 y 13-6-40 de N-P-K, más la aplicación semanal de nitrato de calcio. El manejo de plagas y enfermedades fue preventivo mediante aplicaciones de: Captán ${ }^{\circledR}$, Daconil ${ }^{\circledR}$ o sulfato de cobre para enfermedades, y el uso de extractos vegetales (Viprot ${ }^{\circledR}$, Exakint ${ }^{\circledR}$ y Bio-crack ${ }^{\circledR}$ ) y Confidor ${ }^{\circledR}$ para prevenir plagas. La caracterización agromorfológica se hizo con base en 20 descriptores, algunos de ellos recomendados por el IPGRI (1996), y otros fueron variables de interés agronómico relacionadas con la fenología y producción de frutos.

\section{Análisis estadístico}

Se hicieron análisis de varianza con el modelo lineal de bloques completos al azar, y con los promedios y estandarización de cada variable se efectuó un análisis de componentes principales con la matriz de varianzas y covarianzas, mediante el que se determinaron las variables de mayor valor descriptivo de la variación fenotípica. Con las variables estandarizadas también se hizo un análisis de conglomerados de agrupación jerárquica por el método Ward, donde el punto de corte entre grupos significativamente diferentes, se basó en la significancia del valor de $\mathrm{t}(\mathrm{P} \leq 0.05)$ con ayuda del programa Clustan Graphics (Clustan ${ }^{\circledR}$; Clustan Graphics Version 5.22, Dec. 2001. Clustan Ltd Edinburgh, Scotland).

\section{RESULTADOS Y DISCUSIÓN}

En los análisis de varianza se detectaron diferencias significativas $(P \leq 0.05)$ entre las colectas para todas las variables, excepto para días a fructificación del quinto racimo. Los mayores coeficientes de variación (19.2 a $26.0 \%)$ se obtuvieron en número de flores por racimo, peso medio de frutos, frutos por racimo y total de frutos al quinto racimo (Cuadro 2), que indican alta variabilidad en el comportamiento de las colectas crecidas bajo invernadero.

En el análisis de componentes principales se encontró que con los tres primeros componentes se explica 86.25 $\%$ de la variación fenotípica total. Las variables días a inicio de floración, días a fructificación, días a maduración de frutos, altura de planta a los $30 \mathrm{~d}$ del trasplante, peso del fruto, frutos por racimo y total de frutos al quinto racimo, fueron las características con mayor valor descriptivo de esta variabilidad (Cuadro 3).

Las colectas provenientes de la Costa y Sierra Sur de Oaxaca, llamadas localmente 'Cuatomate', 'Ojo de venado' y otros, se dispersan entre el primero y segundo cuadrantes, en el sentido de las manecillas del reloj (Figura 1), y se distinguieron por presentar valores bajos en días a floración, fructificación y maduración de frutos. Los frutos tipo cereza redondos (tipo 'Cherry') aunque ampliamente dispersos, tendieron a asociarse con mayor altura de planta a los 30 d después del trasplante, mayor número de frutos por racimo y mayor distancia entre racimos florales; es decir, son de crecimiento indeterminado, de ramificación profusa y regularmente ocupan 
grandes áreas de terreno, pero también crecen en cercas, e incluso enredadas entre arbustos o árboles. En la Figura 1 se muestra la tendencia de diferenciación morfológica entre las colectas de tomates semidomesticados y silvestres.

Cuadro 1. Listado de muestras poblacionales evaluadas de tomates silvestres y semidomesticados de Oaxaca, México.

\begin{tabular}{|c|c|c|c|c|c|c|}
\hline Población & $\begin{array}{c}\text { Localidad, paraje o sitio de } \\
\text { muestreo }\end{array}$ & Municipio & Latitud N & Longitud $\mathrm{O}$ & $\begin{array}{l}\text { Altitud } \\
(\mathrm{m})\end{array}$ & $\begin{array}{c}\text { Origen de la } \\
\text { muestra }\end{array}$ \\
\hline CIIDIR-02 & San Juan las Huertas & Putla Villa de Guerrero & $16^{\circ} 54^{\prime} 59^{\prime \prime}$ & $97^{\circ} 56^{\prime} 06^{\prime \prime}$ & 578 & Semidomesticado \\
\hline CIIDIR-21 & Villa Hidalgo Yalalag & Villa Hidalgo Yalalag & $17^{\circ} 11^{\prime} 05^{\prime \prime}$ & $96^{\circ} 10^{\prime} 40^{\prime \prime}$ & 1240 & Silvestre \\
\hline CIIDIR-22 & Villa Hidalgo Yalalag & Villa Hidalgo Yalalag & $17^{\circ} 11^{\prime} 05^{\prime \prime}$ & $96^{\circ} 10^{\prime} 40^{\prime \prime}$ & 1240 & Semidomesticado \\
\hline CIIDIR-23 & Puerto Escondido & San Pedro Mixtepec & $15^{\circ} 51^{\prime} 43^{\prime \prime}$ & $97^{\circ} 04^{\prime} 18^{\prime \prime}$ & 60 & Silvestre \\
\hline CIIDIR-24 & Villa Hidalgo Yalalag & Villa Hidalgo Yalalag & $17^{\circ} 11^{\prime} 05^{\prime \prime}$ & $96^{\circ} 10^{\prime} 40^{\prime \prime}$ & 1240 & Semidomesticado \\
\hline CIIDIR-27 & La Reforma & Acatlán de Pérez Figueroa & $18^{\circ} 29^{\prime} 06^{\prime \prime}$ & $96^{\circ} 27^{\prime} 54^{\prime \prime}$ & 96 & Semidomesticado \\
\hline CIIDIR-29 & Pueblo Viejo & San José Chiltepec & $17^{\circ} 54^{\prime} 31^{\prime \prime}$ & $96^{\circ} 03^{\prime} 26^{\prime \prime}$ & 70 & Semidomesticado \\
\hline CIIDIR-30 & Santa Cruz Xoxocotlán & Santa Cruz Xoxocotlán & $17^{\circ} 03^{\prime} 49^{\prime \prime}$ & $96^{\circ} 43^{\prime}$ & 1530 & Silvestre \\
\hline CIIDIR-31 & San Pedro y San Pablo Ayutla & San Pedro y San Pablo Ayutla & $17^{\circ} 01^{\prime} 32^{\prime \prime}$ & $96^{\circ} 04^{\prime} 48^{\prime \prime}$ & 2020 & Silvestre \\
\hline CIIDIR-34 & Chalcatongo de Hidalgo & Chalcatongo de Hidalgo & $17^{\circ} 01^{\prime} 45^{\prime \prime}$ & $97^{\circ} 34^{\prime} 10^{\prime \prime}$ & 2300 & Semidomesticado \\
\hline CIIDIR-38 & Santa Cruz Xoxocotlán & Santa Cruz Xoxocotlán & $17^{\circ} 03^{\prime} 49^{\prime \prime}$ & $96^{\circ} 43^{\prime}$ & 1530 & Silvestre \\
\hline CIIDIR-39 & Santo Domingo Albarradas & Santo Domingo Albarradas & $17^{\circ} 04^{\prime}$ & $96^{\circ} 12^{\prime}$ & 1480 & Silvestre \\
\hline CIIDIR-45 & Santa Cruz Xoxocotlán & Santa Cruz Xoxocotlán & $17^{\circ} 03^{\prime} 49^{\prime \prime}$ & $96^{\circ} 43^{\prime}$ & 1608 & Silvestre \\
\hline CIIDIR-47 & San Francisco Cajonos & San Francisco Cajonos & $17^{\circ} 10^{\prime} 15^{\prime \prime}$ & $96^{\circ} 15^{\prime}$ & 1700 & Silvestre \\
\hline CIIDIR-48 & San Pablo Yaganiza & San Pablo Yaganiza & $17^{\circ} 08^{\prime} 43^{\prime \prime}$ & $96^{\circ} 13^{\prime} 40^{\prime \prime}$ & 1450 & Silvestre \\
\hline CIIDIR-49 & Ayotzintepec & Ayotzintepec & $17^{\circ} 40^{\prime} 25^{\prime \prime}$ & $96^{\circ} 07^{\prime} 40^{\prime \prime}$ & 120 & Semidomesticado \\
\hline CIIDIR-51 & Chicalito & Santa María Jacatepec & $17^{\circ} 51^{\prime} 36^{\prime \prime}$ & $96^{\circ} 12^{\prime} 41^{\prime}$ & 47 & Semidomesticado \\
\hline CIIDIR-52 & San Pedro Ixcatlán & San Pedro Ixcatlán & $18^{\circ} 08^{\prime} 41^{\prime \prime}$ & $96^{\circ} 30^{\prime} 36^{\prime \prime}$ & 101 & Semidomesticado \\
\hline CIIDIR-53 & La Reforma & Ayotzintepec & $17^{\circ} 40^{\prime} 25^{\prime \prime}$ & $96^{\circ} 07^{\prime} 40^{\prime \prime}$ & 120 & Semidomesticado \\
\hline CIIDIR-57 & Puerto Escondido & San Pedro Mixtepec & $15^{\circ} 51^{\prime} 43^{\prime \prime}$ & $97^{\circ} 04^{\prime} 18^{\prime \prime}$ & 60 & Silvestre \\
\hline CIIDIR-58 & Santa María Guienagati & Santa María Guienagati & $16^{\circ} 44^{\prime} 15^{\prime \prime}$ & $95^{\circ} 21^{\prime} 15^{\prime \prime}$ & 400 & Semidomesticado \\
\hline CIIDIR-60 & Chayotepec & Santa María Guienagati & $16^{\circ} 44^{\prime} 35^{\prime \prime}$ & $95^{\circ} 28^{\prime} 33^{\prime \prime}$ & 1540 & Silvestre \\
\hline CIIDIR-62 & San Blas Atempa & San Blas Atempa & $16^{\circ} 19^{\prime} 35^{\prime \prime}$ & $95^{\circ} 13^{\prime} 32^{\prime \prime}$ & 40 & Semidomesticado \\
\hline CIIDIR-63 & San Blas Atempa & San Blas Atempa & $16^{\circ} 19^{\prime} 35^{\prime \prime}$ & $95^{\circ} 13^{\prime} 32^{\prime \prime}$ & 40 & Semidomesticado \\
\hline CIIDIR-64 & San Blas Atempa & San Blas Atempa & $16^{\circ} 19^{\prime} 35^{\prime \prime}$ & $95^{\circ} 13^{\prime} 32^{\prime \prime}$ & 40 & Semidomesticado \\
\hline CIIDIR-65 & Santa Cruz Xoxocotlán & Santa Cruz Xoxocotlán & $17^{\circ} 03^{\prime} 49^{\prime \prime}$ & $96^{\circ} 43^{\prime}$ & 1530 & Silvestre \\
\hline CIIDIR-69 & Vigastepec & Teotitlán de Flores Magón & $18^{\circ} 10^{\prime} 30^{\prime \prime}$ & $97^{\circ} 02^{\prime} 13^{\prime \prime}$ & 1980 & Semidomesticado \\
\hline CIIDIR-70 & San Martín Toxpalán & San Martín Toxpalán & $18^{\circ} 06^{\prime} 00^{\prime \prime}$ & $97^{\circ} 03^{\prime} 26^{\prime \prime}$ & 1050 & Silvestre \\
\hline CIIDIR-72 & San Blas Atempa & San Blas Atempa & $16^{\circ} 19^{\prime} 35^{\prime \prime}$ & $95^{\circ} 13^{\prime} 32^{\prime \prime}$ & 40 & Semidomesticado \\
\hline CIIDIR-73 & San Blas Atempa & San Blas Atempa & $16^{\circ} 19^{\prime} 35^{\prime \prime}$ & $95^{\circ} 13^{\prime} 32^{\prime \prime}$ & 40 & Silvestre \\
\hline CIIDIR-74 & Plan de Zaragoza & Santiago Nuyoó & $16^{\circ} 57^{\prime} 30^{\prime \prime}$ & $97^{\circ} 44^{\prime} 51^{\prime \prime}$ & 1515 & Silvestre \\
\hline CIIDIR-76 & San Blas Atempa & San Blas Atempa & $16^{\circ} 19^{\prime} 35^{\prime \prime}$ & $95^{\circ} 13^{\prime} 32^{\prime \prime}$ & 40 & Semidomesticado \\
\hline CIIDIR-77 & San Blas Atempa & San Blas Atempa & $16^{\circ} 19^{\prime} 35^{\prime \prime}$ & $95^{\circ} 13^{\prime} 32^{\prime \prime}$ & 40 & Silvestre \\
\hline CIIDIR-78 & San Blas Atempa & San Blas Atempa & $16^{\circ} 19^{\prime} 35^{\prime \prime}$ & $95^{\circ} 13^{\prime} 32^{\prime \prime}$ & 40 & Silvestre \\
\hline CIIDIR-79 & San Blas Atempa & San Blas Atempa & $16^{\circ} 19^{\prime} 35^{\prime \prime}$ & $95^{\circ} 13^{\prime} 32^{\prime \prime}$ & 40 & Silvestre \\
\hline CIIDIR-80 & El Camarón & Nejapa de Madero & $16^{\circ} 33^{\prime} 15^{\prime \prime}$ & $96^{\circ} 01^{\prime} 37^{\prime}$ & 690 & Silvestre \\
\hline CIIDIR-82 & Santiago Cuixtla & Santos Reyes Nopala & $16^{\circ} 03^{\prime} 40^{\prime \prime}$ & $97^{\circ} 11^{\prime} 07^{\prime \prime}$ & 468 & Silvestre \\
\hline CIIDIR-83 & Santiago Cuixtla & Santos Reyes Nopala & $16^{\circ} 03^{\prime} 40^{\prime \prime}$ & $97^{\circ} 11^{\prime} 07^{\prime \prime}$ & 468 & Silvestre \\
\hline CIIDIR-84 & Santiago Cuixtla & Santos Reyes Nopala & $16^{\circ} 03^{\prime} 40^{\prime \prime}$ & $97^{\circ} 11^{\prime} 07^{\prime}$ & 468 & Semidomesticado \\
\hline CIIDIR-88 & Santa Lucía Teotepec & Santos Reyes Nopala & $16^{\circ} 08^{\prime} 35^{\prime \prime}$ & $97^{\circ} 12^{\prime} 22^{\prime \prime}$ & 1210 & Silvestre \\
\hline CIIDIR-89 & El Carrizal & Santa Cruz Zenzontepec & $16^{\circ} 31^{\prime} 56^{\prime \prime}$ & $97^{\circ} 26^{\prime} 33^{\prime \prime}$ & 1040 & Silvestre \\
\hline CIIDIR-90 & El Carrizal & Santa Cruz Zenzontepec & $16^{\circ} 31^{\prime} 56^{\prime \prime}$ & $97^{\circ} 26^{\prime} 33^{\prime \prime}$ & 1040 & Silvestre \\
\hline CIIDIR-94 & Puerto Escondido & San Pedro Mixtepec & $15^{\circ} 51^{\prime} 43^{\prime \prime}$ & $97^{\circ} 04^{\prime} 18^{\prime \prime}$ & 60 & Semidomesticado \\
\hline CIIDIR-97 & San José Chacalapa & San Pedro Pochutla & $15^{\circ} 49^{\prime} 43^{\prime \prime}$ & $96^{\circ} 27^{\prime} 51^{\prime \prime}$ & 230 & Silvestre \\
\hline CIIDIR-98 & Santa María Colotepec & Santa María Colotepec & $15^{\circ} 53^{\prime} 50^{\prime \prime}$ & $96^{\circ} 56^{\prime} 15^{\prime \prime}$ & 50 & Silvestre \\
\hline CIIDIR-100 & Puerto Escondido & San Pedro Mixtepec & $15^{\circ} 51^{\prime} 43^{\prime \prime}$ & $97^{\circ} 04^{\prime} 18^{\prime \prime}$ & 60 & Silvestre \\
\hline CIIDIR-101 & Reyes & San Pedro Pochutla & $15^{\circ} 48^{\prime} 48^{\prime \prime}$ & $96^{\circ} 29^{\prime} 16^{\prime \prime}$ & 220 & Semidomesticado \\
\hline CIIDIR-102 & Santa María Mixtepec & San Gabriel Mixtepec & $16^{\circ} 01^{\prime} 26^{\prime \prime}$ & $97^{\circ} 03^{\prime} 07^{\prime \prime}$ & 590 & Silvestre \\
\hline CIIDIR-104 & El Tomatal & Santa María Colotepec & $15^{\circ} 47^{\prime} 05^{\prime \prime}$ & $96^{\circ} 55^{\prime} 14^{\prime \prime}$ & 15 & Semidomesticado \\
\hline
\end{tabular}


Cuadro 2. Promedios, coeficientes de variación (CV) y cuadrados medios (CM) de cada variable evaluada en tomates silvestres y semidomesticados de Oaxaca, México. Primavera 2009.

\begin{tabular}{|c|c|c|c|}
\hline Variables & Promedio & $\mathrm{CV}(\%)$ & $\mathrm{CM}$ \\
\hline Días a inicio de floración ${ }^{\dagger}$ & 21.8 & 8.9 & $27.8^{* *}$ \\
\hline Días a floración del quinto racimo ${ }^{\dagger}$ & 44.6 & 5.8 & $28.7 * *$ \\
\hline Días a inicio de fructificación ${ }^{\dagger}$ & 31.5 & 8.1 & $24.8^{* *}$ \\
\hline Días a fructificación del quinto racimo ${ }^{\dagger}$ & 54.2 & 6.0 & $11.5^{\mathrm{ns}}$ \\
\hline Días a inicio de maduración de frutos ${ }^{\dagger}$ & 63.1 & 4.0 & $40.2 * *$ \\
\hline Días a maduración en el quinto racimo ${ }^{\dagger}$ & 52.2 & 6.3 & $72.0^{* *}$ \\
\hline Altura de planta a $30 \mathrm{~d}$ de trasplante $^{\dagger}(\mathrm{cm})$ & 26.5 & 15.9 & $174.9 * *$ \\
\hline Altura de planta a $60 \mathrm{~d}$ del trasplante $\mathrm{e}^{\dagger}(\mathrm{m})$ & 1.5 & 8.1 & $753.9 * *$ \\
\hline Número promedio de foliolos de una hoja madura & 7.7 & 7.9 & $0.9 * *$ \\
\hline Longitud de una hoja madura sin pedúnculo $(\mathrm{cm})$ & 34.7 & 9.0 & $50.1^{* *}$ \\
\hline Diámetro de tallo $(\mathrm{cm})$ & 1.0 & 11.9 & $0.1 * *$ \\
\hline Distancia de entrenudos foliares $(\mathrm{cm})$ & 6.6 & 14.2 & $1.4^{*}$ \\
\hline Distancia entre racimos florales $(\mathrm{cm})$ & 18.3 & 14.1 & $14.8^{* *}$ \\
\hline Número de flores por racimo & 6.5 & 19.2 & $3.5^{* *}$ \\
\hline Total de frutos por planta al $5^{\circ}$ racimo & 29.1 & 26.0 & $301.7 * *$ \\
\hline Diámetro de fruto $(\mathrm{cm})$ & 2.3 & 7.0 & $1.7 * *$ \\
\hline Longitud de fruto $(\mathrm{cm})$ & 2.2 & 7.8 & $1.9^{* *}$ \\
\hline Número de lóculos por fruto & 2.2 & 9.3 & $0.8^{* *}$ \\
\hline Peso de fruto $(\mathrm{g})$ & 8.3 & 25.9 & $166.4^{* *}$ \\
\hline Número de frutos por racimo & 5.8 & 26.0 & $12.1^{* *}$ \\
\hline
\end{tabular}

${ }^{\top}$ Después del trasplante; $\mathrm{ns}=$ significativamente diferentes $(\mathrm{P}>0.05) ; * \mathrm{y} * *$ Significativamente diferentes a nivel de $\mathrm{P} \leq 0.05$ y $\mathrm{P} \leq 0.01$, respectivamente.

Cuadro 3. Vectores y valores propios del análisis de componentes principales (CP) con las variables de mayor valor descriptivo de la varianza total, en tomates silvestres y semidomesticados de Oaxaca, México.

\begin{tabular}{lccc}
\hline Variables agromorfológica & CP1 & CP2 & CP3 \\
\hline Días a inicio de floración después del trasplante & 0.450 & 0.170 & -0.167 \\
Días a inicio de fructificación después del trasplante & 0.486 & 0.151 & -0.078 \\
Días a inicio de maduración de frutos después del trasplante & 0.439 & 0.282 & 0.096 \\
Días a maduración del 5 ${ }^{\circ}$ racimo después del trasplante & 0.351 & 0.304 & 0.273 \\
Altura de planta a 30 d del trasplante & -0.360 & 0.329 & 0.326 \\
Distancia entre racimos florales & -0.159 & 0.282 & 0.520 \\
Total de frutos al quinto racimo & -0.198 & 0.420 & -0.487 \\
Peso medio de frutos & -0.106 & 0.484 & 0.175 \\
Número de frutos por racimo & -0.198 & 0.484 & 0.175 \\
Valores propios & $\mathbf{3 . 8 0}$ & $\mathbf{2 . 5 7}$ & $\mathbf{1 . 6 0}$ \\
Varianza explicada (\%) & $\mathbf{3 9 . 9 6}$ & $\mathbf{2 8 . 5 1}$ & $\mathbf{1 7 . 7 7}$ \\
Varianza acumulada (\%) & $\mathbf{3 9 . 9 6}$ & $\mathbf{6 8 . 4 8}$ & $\mathbf{8 6 . 2 5}$ \\
\hline
\end{tabular}

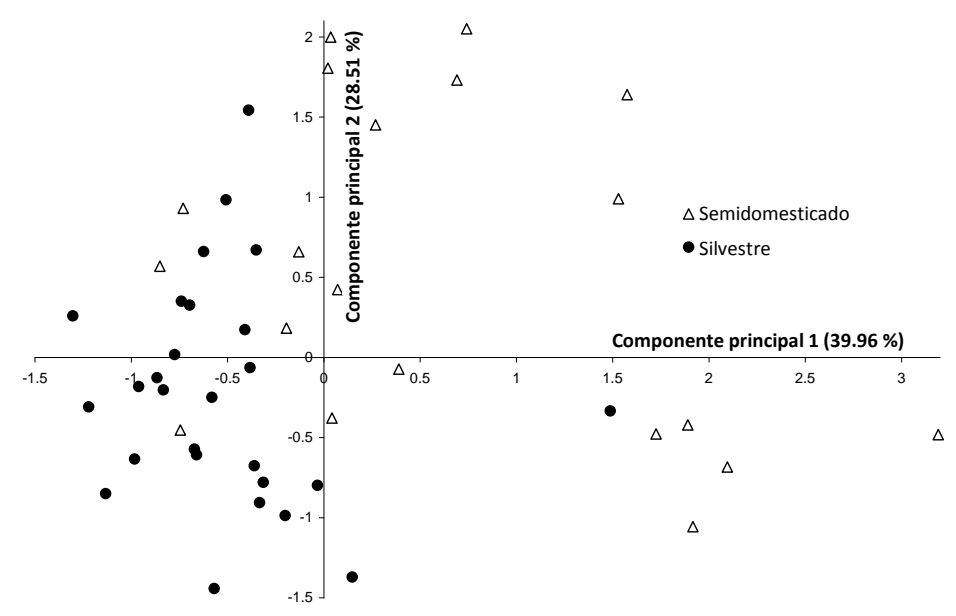

Figura 1. Dispersión de 49 colectas de tomates silvestres y semidomesticados de Oaxaca, con base en los dos primeros componentes principales. 
En el análisis de conglomerados se determinaron ocho subgrupos diferentes $(\mathrm{t}=5.71, \mathrm{gl}=47, \mathrm{P} \leq 0.05)$, integrados en dos grandes grupos morfológicos dentro de la variedad cerasiforme (Figura 2); el primero corresponde a los tomates semidomesticados, con mayor tamaño de fruto $(2.8$ a $3.7 \mathrm{~cm}$ de diámetro $)$, que probablemente se cruzaron con variedades locales por presentar formas de fruto en forma de riñón con hombros u oblongo-elongado (forma de pera), o bien son producto de selección realizada por el agricultor. El segundo grupo correspondió a cerasiforme silvestre con frutos pequeños de hasta $1.8 \mathrm{~cm}$ de diámetro, con dos lóculos y un peso medio no mayor de $3 \mathrm{~g}$. Álvarez-Hernández et al. (2009) también describieron patrones semejantes en tomates silvestres de Michoacán; de 2.1 a $2.4 \mathrm{~cm}$ en los frutos más grandes y de $1 \mathrm{a} 1.1 \mathrm{~cm}$ en los pequeños, en evaluaciones de campo.

Dentro de cada gran grupo de tomate se diferenciaron cuatro subgrupos significativamente diferentes (Cuadro 4). Entre los subgrupos semidomesticados (I, II, III, IV), las diferencias se presentaron en días a inicio de floración y floración del quinto racimo (el subgrupo IV fue el más tardío, y los subgrupos I y II fueron precoces), altura a los $30 \mathrm{~d}$ del trasplante, número de frutos al quinto racimo, número de frutos por racimo y peso medio de frutos. Entre los subgrupos de silvestres (V, VI, VII, VIII) las diferencias se determinaron en días a floración, fructificación y maduración, distancia entre racimos florales, longitud de entrenudos foliares, altura a los $30 \mathrm{y}$
$60 \mathrm{~d}$, mientras que en características de frutos fueron semejantes (Cuadro 4).

Esta variación agromorfológica del tomate clasificada en ocho grupos diferentes, proporciona una idea general de la variación de esta especie en Oaxaca. La clasificación en dos grandes grupos, poblaciones semidomesticadas y poblaciones silvestres con tamaños y pesos de fruto distintos (Figura 2), permite inferir que están ocurriendo dos procesos evolutivos del tomate en Oaxaca como resultado del manejo o no del hombre. En las poblaciones semidomesticadas los frutos fueron esféricos, periformes o achatados con hombros de 3 a 5 lóculos, diámetros de 2.5 a $3.7 \mathrm{~cm}$ y longitudes de 2.6 a $3.1 \mathrm{~cm}$, con alta producción de flores por racimo (5 a 8 ) y frutos al quinto racimo (21 a 46), precocidad a la fructificación del quinto racimo (53 a $57 \mathrm{~d}$ después del trasplante) y crecimiento indeterminado (1.5 a $1.8 \mathrm{~m}$ a $60 \mathrm{~d}$ del trasplante). En el grupo de poblaciones silvestre los frutos fueron más pequeños, indicativo de su condición silvestre, con dos lóculos, diámetros y longitudes menores a $2 \mathrm{~cm}$, alta producción de flores por racimo ( 5 a 7 ) y abundantes frutos al quinto racimo (20 a 30), precocidad a la fructificación del quinto racimo (53 a $55 \mathrm{~d}$ después del trasplante) y crecimiento indeterminado (1.3 a $1.7 \mathrm{~m}$ a 60 d del trasplante). Los valores de estas características indican una condición silvestre y son argumentos para establecer que su preservación in situ continúa sin la intervención del hombre.

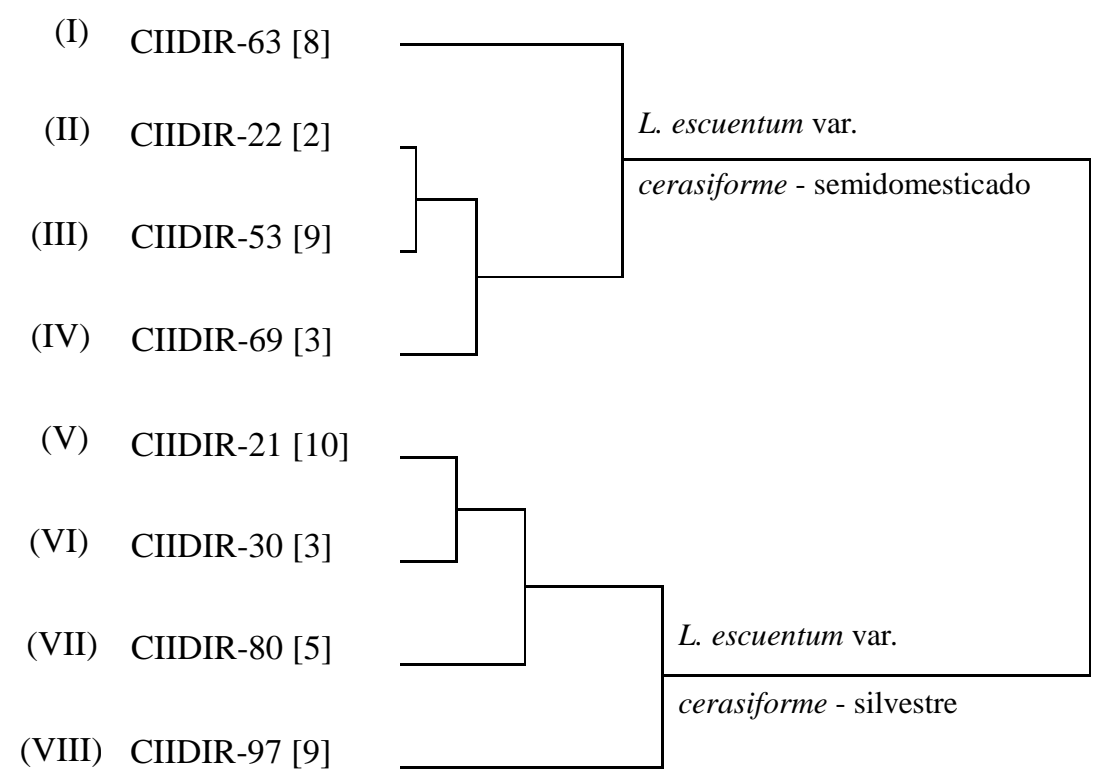

Figura 2. Dendrograma de ocho grupos significativamente diferentes de 49 colectas de tomates silvestres y semidomesticados de Oaxaca, México; [n] representa el número de colectas en cada grupo. 
Cuadro 4. Promedios de la variación agromorfológica de ocho grupos formados en el análisis de conglomerados de 49 colectas de tomates silvestres y semidomesticados de Oaxaca, México.

\begin{tabular}{|c|c|c|c|c|c|c|c|c|}
\hline \multirow{2}{*}{ Variables agromorfológicas } & \multicolumn{8}{|c|}{ Grupos fenotípicos ( $\mathrm{n}=$ número de colectas) } \\
\hline & $\mathrm{I}(8)$ & II (2) & III (9) & IV (3) & $\mathrm{V}(10)$ & VI (3) & VII (5) & VIII (9) \\
\hline Días a inicio de floración ${ }^{\dagger}$ & 20.8 & 22.0 & 21.6 & 28.1 & 21.3 & 16.8 & 19.5 & 24.2 \\
\hline Días a floración del $5^{\circ}$ racimo ${ }^{\dagger}$ & 43.8 & 42.7 & 45.5 & 50.2 & 44.3 & 45.3 & 38.9 & 46.3 \\
\hline Días a inicio de fructificación ${ }^{\dagger}$ & 30.1 & 30.2 & 31.7 & 39.2 & 31.0 & 28.2 & 29.0 & 33.1 \\
\hline Días a fructificación del $5^{\circ}$ racimo $^{\dagger}$ & 53.9 & 53.0 & 54.7 & 57.3 & 53.7 & 52.7 & 53.8 & 54.4 \\
\hline Días a inicio de maduración ${ }^{\dagger}$ & 62.9 & 63.8 & 63.4 & 71.0 & 62.7 & 60.3 & 56.3 & 65.1 \\
\hline Días a maduración del $5^{\circ}$ racimo $^{\dagger}$ & 52.7 & 50.5 & 52.8 & 62.3 & 52.1 & 52.3 & 41.5 & 54.1 \\
\hline Altura de planta a $30 \mathrm{~d}^{\dagger}(\mathrm{cm})$ & 37.3 & 36.8 & 26.8 & 20.4 & 24.2 & 34.6 & 21.7 & 19.0 \\
\hline Altura de planta a $60 \mathrm{~d}^{\dagger}(\mathrm{cm})$ & 1.5 & 1.8 & 1.5 & 1.5 & 1.6 & 1.7 & 1.6 & 1.3 \\
\hline Núm. de foliolos por hoja & 7.4 & 7.9 & 8.1 & 7.9 & 7.7 & 6.9 & 7.5 & 7.9 \\
\hline Longitud de hoja & 35.6 & 37.6 & 38.3 & 38.6 & 33.3 & 23.3 & 35.2 & 33.3 \\
\hline Diámetro tallo $(\mathrm{cm})$ & 1.1 & 1.2 & 1.2 & 1.1 & 0.9 & 0.6 & 0.9 & 0.9 \\
\hline Long. entrenudos foliares & 6.8 & 6.9 & 6.7 & 7.2 & 6.7 & 7.7 & 6.4 & 5.8 \\
\hline Distancia entre racimos florales & 19.3 & 20.5 & 18.7 & 18.6 & 19.2 & 21.4 & 16.2 & 15.6 \\
\hline Núm. de flores por racimo & 7.6 & 4.9 & 6.2 & 5.6 & 6.9 & 6.6 & 5.3 & 6.5 \\
\hline Núm. total de frutos al $5^{\circ}$ racimo & 46.3 & 21.6 & 25.5 & 28.6 & 27.6 & 20.5 & 30.0 & 23.2 \\
\hline Diámetro de fruto & 3.2 & 3.7 & 2.8 & 2.5 & 1.7 & 1.4 & 1.7 & 1.8 \\
\hline Longitud de fruto & 3.1 & 2.8 & 3.0 & 2.6 & 1.5 & 1.4 & 1.6 & 1.6 \\
\hline Núm. de lóculos por fruto & 2.2 & 4.4 & 2.3 & 2.3 & 2.0 & 2.0 & 2.1 & 2.0 \\
\hline Peso medio deL fruto $(\mathrm{g})$ & 18.1 & 19.4 & 13.3 & 10.6 & 2.7 & 1.5 & 2.3 & 3.0 \\
\hline Núm. de frutos por racimo & 9.3 & 4.3 & 5.1 & 5.7 & 5.5 & 4.1 & 6.0 & 4.6 \\
\hline
\end{tabular}

†'ías después del trasplante

\section{CONCLUSIONES}

La colección de 49 muestras poblacionales de tomate, silvestres y semidomesticadas, de Oaxaca presentaron diferencias significativas en 19 características fenológicas y morfológicas de planta, tallo, hoja, flores y frutos. Las colectas fueron agrupadas en dos grandes grupos; cuatro subgrupos de poblaciones semidomesticadas y cuatro subgrupos de silvestres, con diferencias importantes en tamaño y peso de frutos, entre otras características. Las poblaciones semidomesticadas presentaron variación de 2.5 a $3.7 \mathrm{~cm}$ de diámetro y de 10.6 a $19.4 \mathrm{~g}$ por fruto, y las silvestres de 1.4 a $1.8 \mathrm{~cm}$ y de 1.5 a $3.0 \mathrm{~g}$ de diámetro $\mathrm{y}$ peso de fruto, respectivamente.

\section{BIBLIOGRAFÍA}

Álvarez-Hernández J C, H Cortez-Madrigal, I García-Ruiz (2009) Exploración y caracterización de poblaciones silvestres de jitomate (Solanaceae) en tres regiones de Michoacán, México. Polibotánica 28:139-159.

IPGRI, Instituto Internacional de Recursos Fitogenéticos (1996) Descriptores de tomate (Lycopersicon spp.). Instituto Internacional de Recursos Fitogenéticos, Roma, Italia. 49 p.

Jenkins J A (1948) The origin of the cultivated tomato. Econ. Bot. 2:379-392.

Molina M J C, L Córdova T (2006) Conservación ex situ. In: Recursos Fitogenéticos de México para la Alimentación y la Agricultura. Informe Nacional 2006. J C Molina M, L Córdova T (eds). Secretaría de Agricultura, Ganadería, Desarrollo Rural, Pesca y Alimentación y Sociedad Mexicana de Fitogenética, A.C. Chapingo, México. pp:59-100.
Nuez F, B Pico (1997) Germplasm of tomato and wild relatives from genebank of the Polytechnic University of Valencia. In: Proc. 1st Intl. Conf. ON the Processing and 1st Intl. Symp. Tropical Tomato Diseases. 18-22 November, Recife, Pernambuco, Brazil. G Alves M, G M B Lopes, C Hayward, R R L Mariano, E A A Marahao (eds). The American Society for Horticultural Science and Empresa Pernambucana de Pesquisa Agropecuária, Pernambuco, Brazil. pp:83-89.

Peralta I E, D M Spooner (2007) History, origin and early cultivation of tomato (Solanaceae). In: Genetic Improvement of Solanaceous Crops. Vol. 2. Tomato. M K Razdan, A K Mattoo (eds). Science Publishers. Enfield, New Hampshire, USA. pp:1-24.

Rick C M, J F Fobes (1975) Allozyme variation in the cultivated tomato and closely related species. Bull. Torrey Bot. Club 102:376-384.

Rick C M (1986) Germplasm resources in the wild tomato species. Acta Hort. 190:39-48

Sánchez-Peña $\mathbf{P}, \quad \mathbf{K}$ Oyama, J Núñez-Farfán, J Fornoni, $\mathbf{S}$ Hernández-Verdugo, J Márquez-Guzmán, J A GarzónTiznado (2006) Sources of resistance of whitefly (Bemisia spp.) in wild populations of Solanum lycopersicum var. cerasiforme (Dunal) Spooner G J, Anderson et R K Jansen, in Northwestern México. Gen. Res. Crop Evol. 53:711-719.

Schauer N, Y Semel, U Roessner, A Gur, I Balbo, F Carrari, T Pleban, A Perez-Melis, C Bruedigam, J Kopka, L Willmitzer, D Zamir, A R Fernie (2006) Comprehensive metabolic profiling and phenotyping of interspecific introgression lines for tomato improvement. Nature Biotechnol. 24:447-454.

Sevilla R, M Holle (2004) Recursos Genéticos Vegetales. Luis León Asociados S.R.L. Lima, Perú. 445 p.

Zamir D, S Grandillo, S D Tanksley (1999) Genes from wild species for the improvement of yield and quality of processing tomatoes. Acta Hort. 487:285-288. 